Homology, Homotopy and Applications, vol. 18(2), 2016, pp.359-375

\title{
MOTION PLANNING IN REAL FLAG MANIFOLDS
}

\author{
JESÚS GONZÁLEZ, BÁRBARA GUTIÉRREZ, DARWIN GUTIÉRREZ \\ AND ADRIANA LARA
}

(communicated by Donald M. Davis)

\begin{abstract}
Starting from Borel's description of the mod-2 cohomology of real flag manifolds, we give a minimal presentation of the cohomology ring of semi-complete flag manifolds $F_{k, m}:=$ $F(1, \ldots, 1, m)$, where 1 is repeated $k$ times. This is used to estimate Farber's topological complexity of $F_{k, m}$ when $m$ approaches (from below) a 2-power. In particular, we get almost sharp estimates for $F_{2,2^{e}-1}$ which resemble the known situation for the real projective spaces $F_{1,2^{e}}$. Our results indicate that the agreement between the topological complexity and the immersion dimension of real projective spaces no longer holds for other flag manifolds. We also get corresponding results for the $s$-th higher topological complexity of these spaces, proving the surprising fact that, as $s$ increases, our cohomological estimates become stronger. Indeed, we get a full description of the higher motion planning problem of some of these manifolds. As a byproduct, we get a complete computation of the higher topological complexity of all closed surfaces (orientable or not).
\end{abstract}

\section{Introduction and main results}

The concept of topological complexity (TC) of a space $X$ was introduced early this millennium by Michael Farber as a way to utilize techniques from homotopy theory in order to model and study, from a topological perspective, the motion planning problem in robotics. If $P(X)$ stands for the space of free paths in $X$, then $\mathrm{TC}(X)$ is the reduced Schwarz genus (also known as sectional category) of the fibration $e: P(X) \rightarrow$ $X \times X$ given by $e(\gamma)=(\gamma(0), \gamma(1))$. We refer the reader to the book $[8]$ and the references therein for a discussion of the meaning, relevance, and basic properties of Farber's concept.

The first author was supported by Conacyt Research Grant 221221. Some portions of this paper are based on the Ph.D. thesis work of the third author at the Mathematics Department of the Escuela Superior de Física y Matemáticas, Instituto Politécnico Nacional. The fourth author was supported by Grant SIP20152082.

Received March 20, 2016, revised April 22, 2016; published on November 18, 2016.

2010 Mathematics Subject Classification: 55M30, 57T15, 68T40, $70 \mathrm{~B} 15$.

Key words and phrases: flag manifold, surface, topological complexity, zero-divisors cup-length, motion planning.

Article available at http://dx.doi.org/10.4310/HHA.2016.v18.n2.a20

Copyright (C) 2016, International Press. Permission to copy for private use granted. 
The idea was generalized a few years later by Rudyak, who defined in $[\mathbf{2 2}]$ the $s$-th topological complexity of $X, \mathrm{TC}_{s}(X)$, as the reduced Schwarz genus of the $s$-th fold evaluation map $e_{s}: P(X) \rightarrow X^{s}$ given by

$$
e_{s}(\gamma)=\left(\gamma(0), \gamma\left(\frac{1}{s-1}\right), \gamma\left(\frac{2}{s-1}\right), \ldots, \gamma\left(\frac{s-2}{s-1}\right), \gamma(1)\right) .
$$

In particular, $\mathrm{TC}=\mathrm{TC}_{2}$. Rudyak's "higher" topological complexity has been studied systematically in $[\mathbf{1}]$.

The purpose of this paper is two fold. For one, we give extensive computations to estimate the value of $\mathrm{TC}_{s}$ on a number of infinite families of semi-complete real flag manifolds $F\left(1^{k}, m\right)$ - the Grassmann type manifolds consisting of $k+1$ tuples $\left(L_{1}, \ldots L_{k}, V\right)$ of mutually orthogonal linear subspaces of $\mathbb{R}^{m+k}$ with $\operatorname{dim}(V)=m$ and $\operatorname{dim}\left(L_{i}\right)=1$ for $1 \leqslant i \leqslant k$. Much of the motivation here comes from an amazing and unexpected connection between Farber's TC and one of the central problems in differential topology, namely the Euclidean immersion dimension for smooth manifolds. Explicitly, for a manifold $M$, let $\operatorname{Imm}(M)$ denote the dimension of the smallest Euclidean space where $M$ can be immersed. Then the main result in [9] asserts that $\mathrm{TC}=\mathrm{Imm}$ for all real projective spaces $\mathbb{R P}^{m}$ except for the only three parallelizable manifolds, $\mathbb{R P} P^{1}, \mathbb{R P}^{3}$, and $\mathbb{R P}^{7}$, for which the relation $\mathrm{TC}=\mathrm{Imm}-1$ holds. Of course, flag manifolds $F\left(1^{k}, m\right)$ are a natural generalization of real projective spaces. So it is natural to ask whether the above relationship between topological complexity and immersion dimension also holds for the larger family of manifolds. Although the Euclidean immersion dimension of real flag manifolds is a much studied problem, and quite a lot of numeric information on it is available to date, the topological complexity of real flag manifolds had not been considered before - except, of course, for the already noted results with real projective spaces. The numeric TC-results in this paper now show that the nice relationship between TC and Imm holding for real projective spaces $F(1, m)$ does not hold for flag manifolds $F\left(1^{k}, m\right)$ with $k>1$. For instance, $F(1,1,1)$ is a closed parallelizable 3 -manifold [16], so that $\operatorname{Imm}(F(1,1,1))=4$. However, Theorem 1.1 below gives $\operatorname{TC}(F(1,1,1)) \in\{5,6\}$. Thus, the relation ' $\mathrm{TC}=\mathrm{Imm}-1$ ' holding for parallelizable real projective spaces no longer holds in the case of the other parallelizable flag manifolds $F\left(1^{k}, 1\right)$. In general, flag manifolds (whether parallelizable of not) seem to have a larger TC than an Imm. For instance, the 7-dimensional flag manifold $F(1,1,3)$ has $\operatorname{Imm}(\mathrm{F}(1,1,3))=10[\mathbf{1 8}, \mathbf{2 3}]$ whereas, according to Theorem 1.1 below, $\operatorname{TC}(F(1,1,3)) \in\{13,14\}$.

The second purpose of this paper aims at exhibiting subtle but substantial differences between Farber's original concept and Rudyak's extended definition. The point is that, after an initial examination, Rudyak's higher TC could seem to be a close relative of Farber's TC. For instance, it has been shown that the families of spaces $X$ whose TC has been computed have an equally computable higher TC (cf. $[\mathbf{1}, \mathbf{1 0}, \mathbf{1 2}])$. Likewise, some theoretical results for TC have reasonable (although sometimes more complicated to prove) higher TC generalizations, see, for instance, $[\mathbf{2}, \mathbf{3}, \mathbf{1 3}, \mathbf{1 9}$. However, other interesting theoretical properties known for TC do not have a known higher TC counterpart. For instance, it is known that the standard upper bound $2 \operatorname{dim}(X)$ for $\mathrm{TC}(X)$ can be lowered by one unit whenever $\pi_{1}(X)=\mathbb{Z}_{2}[\mathbf{4}]$. Before this paper, it was not even clear whether the proof of such a fact could be generalized to the higher TC realm. As a consequence of Theorem 1.2 below (with $k=1$ ), we 
now know that such a potential $\mathrm{TC}_{s}$ generalization is doomed to fail for $s \geqslant 3$.

Closely related to the above fact is the phenomenon that there are infinite families of spaces for which the computation of their TC would require a non-elementary homotopy theoretic argument, but whose higher TC can be computed using purely cohomological methods. Indeed, as shown in this paper (Theorems 1.2 and 1.3), surfaces and flag manifolds $F\left(1^{k}, 2^{e}-k+1\right)$ with $k \leqslant 3$ have such a property. ${ }^{1}$ Technically speaking, this phenomenon can be summarized by saying that, in many cases, the $\mathrm{TC}_{2}$-obstruction described in [4, Theorem 7] vanishes without the vanishing of the analogous $\mathrm{TC}_{s}$-obstruction for $s \geqslant 3$ (see the comments following (2) below).

We next state our main results and explain how they fit within the introductory considerations above. Further comments will be given throughout the paper.

Theorem 1.1 (Corollary 3.14). Let $k$ and $m$ be positive integers, $\delta \in\{0,1, \ldots, k-$ $1\}$, and set $\epsilon=\min (\delta, 1)$ and $\alpha(r)=\max (0, r)$. If e is a nonnegative integer satisfying $2 \delta \leqslant 2^{e} \leqslant m+\delta$, then

$$
(k-\delta+\epsilon)\left(2^{e+1}-1\right)+\alpha\left((\delta-1)\left(2^{e}-1\right)\right)-\epsilon \leqslant \mathrm{TC}\left(F\left(1^{k}, m\right)\right) \leqslant k(2 m+k-1) .
$$

Of course, the parameter $e$ should be taken as large as possible in order to get the full strength of Theorem 1.1. Two special cases (where the estimate in (1) has a gap of a unit) should be singled out from this result, namely:

- $\mathrm{TC}\left(F\left(1,2^{e}\right)\right) \in\left\{2^{e+1}-1,2^{e+1}\right\}$.

- $\mathrm{TC}\left(F\left(1^{2}, 2^{e}-1\right)\right) \in\left\{2^{e+2}-3,2^{e+2}-2\right\}$.

Since $F\left(1,2^{e}\right)$ is the real projective space $\mathbb{R P}^{2^{e}}$, the first situation is resolved by the well known equality

$$
\mathrm{TC}\left(\mathbb{R P}^{2^{e}}\right)=2^{e+1}-1
$$

(see [9]). It might seem reasonable to expect $\operatorname{TC}\left(F\left(1^{2}, 2^{e}-1\right)\right)=2^{e+2}-3$. In any case, proving (disproving) such an equality is equivalent to showing the triviality (nontriviality) of the homotopy obstruction described in [4, Theorem 7] for $X=F\left(1^{2}, 2^{e}-\right.$ 1 ). The relevance of such a task becomes apparent by noticing that, as a special case of Theorem 1.2 below, the $\mathrm{TC}_{s}$-analogue of the above homotopy obstruction does not vanish for these spaces when $s \geqslant 3$ :

Theorem 1.2 (Theorem 4.3). For positive integers $e, k$ and $s$ with $e \geqslant 1+\left\lfloor\frac{k-1}{2}\right\rfloor$ and $k \leqslant 3 \leqslant s, \mathrm{TC}_{s}\left(F\left(1^{k}, 2^{e}-k+1\right)\right)=s \operatorname{dim}\left(F\left(1^{k}, 2^{e}-k+1\right)\right)$.

For instance, when $k=1$, we get $\mathrm{TC}_{s}\left(\mathbb{R P}^{2^{e}}\right)=2^{e} s$ if $s \geqslant 3$ and $e \geqslant 1$, which is certainly not the case for $s=2$, as noted in (2). The restriction $e \geqslant 1$ is also needed as $\mathrm{TC}_{s}\left(S^{1}\right)=s-1$ is well known [22, Section 4].

It should be noted that Theorem 1.2 can be thought of as a very distinguished manifestation of a more general phenomenon, namely: for fixed $k$ and $m$, the mod-2 cohomological estimates in this paper for $\mathrm{TC}_{s}\left(F\left(1^{k}, m\right)\right)$ become sharper as $s$ increases. Such a point will be clarified and worked out in Section 4 of this paper (Remark 4.2 and Corollary 4.8).

Other interesting (almost-sharp) estimates for the higher topological complexity of some semi-complete flag manifolds not considered in Theorem 1.2 are discussed

\footnotetext{
${ }^{1}$ It might be the case that the restriction on $k$ can be removed - see the second half of Remark 4.6.
} 
in Section 4. All together, our results seem to point out to what could be the best estimate that purely cohomological methods can yield for the higher topological complexity of semi-complete flag manifolds $F\left(1^{k}, m\right)$ (see Remark 4.14).

Theorems 1.1 and 1.2 (and related results discussed in Section 4) are based on the identification of suitably long products of zero-divisors. The form of the required factors follows patterns that depend strongly on the value of $k$. The identification of such patterns is a major task in this paper that has greatly benefitted from the help of extensive computer calculations. On the other hand, the complexity of the calculations supporting Theorems 1.1 and 1.2 is in sharp contrast with the easy situation for the complex analogues $F_{\mathbb{C}}\left(n_{1}, \ldots, n_{\ell}\right)$. The latter manifolds are 1-connected and symplectic (even Kähler), so their $s$-th topological complexity is well known (and easy to see) to agree with $s\left(\operatorname{dim}\left(F_{\mathbb{C}}\left(n_{1}, \ldots, n_{\ell}\right)\right)\right) / 2($ see $[\mathbf{1}$, Corollary 3.15$])$.

The final section of the paper deals with the proof of:

Theorem 1.3 (Proposition 5.1). Let $S$ be a closed surface (orientable or not) other than the sphere and the torus. Then $\mathrm{TC}_{s}(S)=2 s$ provided $s \geqslant 3$.

It is folklore that the conclusion of Theorem 1.3 holds true for $s=2$ if $S$ is orientable and has genus at least 2. What is most interesting is to compare Theorem 1.3 with the fact that the precise value of Farber's topological complexity of non-orientable surfaces of genus at least 2 has become an intriguing open question in the field. ${ }^{2}$

Theorem 1.3, which will be a relatively easy consequence of the calculations supporting Theorem 1.2, gives of course an infinite family of spaces for which, just as for the flag manifolds in Theorem 1.2, the $\mathrm{TC}_{s}$ accessibility contrasts with the hardness of the $\mathrm{TC}_{2}$ situation. It would be interesting to know if such a phenomenon holds for other families of spaces.

\section{Cohomology and LS-category of $F\left(1^{k}, m\right)$}

Unless otherwise noted, all cohomology rings we deal with have $\mathbb{F}_{2}$-coefficients. For $i \geqslant 0$, let $e_{i}$ denote the $i$-th elementary symmetric polynomial, and $h_{i}$ denote the $i$-th complete symmetric polynomial $\left(e_{0}=h_{0}=1\right)$. In both cases the relevant variables will be explicitly indicated.

Proposition 2.1. Let $m \geqslant 1$. A minimal presentation for the ring $H^{*}\left(F\left(1^{k}, m\right)\right)$ is given by generators $x_{i}, 1 \leqslant i \leqslant k$, all of dimension 1 , subject to the relations

$$
h_{m+i}\left(x_{1}, \ldots, x_{k+1-i}\right)=0, \quad 1 \leqslant i \leqslant k .
$$

A graded additive basis for $H^{*}\left(F\left(1^{k}, m\right)\right)$ is given by the monomials

$$
x\left(n_{1}, \ldots, n_{k}\right):=\prod_{i=1}^{k} x_{i}^{n_{i}},
$$

where $n_{i} \leqslant m+k-i$, for $i=1, \ldots, k$.

\footnotetext{
${ }^{2}$ There is an argument in the recent preprint [6] for the equality $\mathrm{TC}_{2}\left(N_{g}\right)=4$ for $g \geqslant 4$.
} 
Remark 2.2. The above presentation is a strong generalization of the one given in $[\mathbf{1 4}$, Example 9.5.17] for complete flags - the latter one is not minimal. The direct proof below should be compared to [21], a paper devoted to the proof (using Gröbner bases) of Proposition 2.1.

Proof of Proposition 2.1. For $i=1, \ldots, k+1$, let $\gamma_{i}$ stand for the $i$-th tautological bundle on $F\left(1^{k}, m\right)$, and set $x_{i}=w_{1}\left(\gamma_{i}\right)$ for $i \leqslant k$, and $w_{j}=w_{j}\left(\gamma_{k+1}\right)$ for $j \geqslant 0$, the Stiefel-Whitney classes. Borel's (non-minimal) presentation of $H^{*}\left(F\left(1^{k}, m\right)\right)$ has generators $x_{i}$ and $w_{j}$ with the single (non-homogeneous) relation

$$
\sum_{j \geqslant 0} w_{j} \prod_{i=1}^{k}\left(1+x_{i}\right)=1 .
$$

This expression's component in dimension $j>0$ is

$$
\sum_{0 \leqslant t \leqslant j} w_{j-t} e_{t}\left(x_{1}, \ldots, x_{k}\right)=0 .
$$

In particular, for $j=1$, we get $w_{1}=e_{1}\left(x_{1}, \ldots, x_{k}\right)=h_{1}\left(x_{1}, \ldots, x_{k}\right)$. Assuming inductively that $w_{\ell}=h_{\ell}\left(x_{1}, \ldots, x_{k}\right)$ for $\ell<j,(5)$ gives

$$
w_{j}=\sum_{1 \leqslant t \leqslant j} h_{j-t}\left(x_{1}, \ldots, x_{k}\right) e_{t}\left(x_{1}, \ldots, x_{k}\right)=h_{j}\left(x_{1}, \ldots, x_{k}\right) .
$$

This uses the basic relation between elementary and complete symmetric polynomials

$$
\sum_{t=0}^{j}(-1)^{t} e_{t}\left(x_{1}, \ldots, x_{k}\right) h_{j-t}\left(x_{1}, \ldots, x_{k}\right)=0 .
$$

Therefore, the generators $w_{j}$ are superfluous and, since $w_{j}=0$ for $j>m$, we get $h_{m+i}\left(x_{1}, \ldots, x_{k}\right)=0$ for $i>0$. This is (3) if $i=1$, otherwise use

$$
h_{m+i}\left(x_{1}, \ldots, x_{k}\right)=h_{m+i}\left(x_{1}, \ldots, x_{k-1}\right)+x_{k} h_{m+i-1}\left(x_{1}, \ldots, x_{k}\right)
$$

to get $h_{m+i}\left(x_{1}, \ldots, x_{k-1}\right)=0$ for $i>1$. Iteration of this argument yields (3). Further, these equations can be used to write any power $x_{i}^{\ell}$ with $\ell>m+k-i$ in terms of powers $x_{j}^{n}$ with $j<i$ or $n<\ell$. This shows that the monomials in (4) are additive generators of $H^{*}\left(F\left(1^{k}, m\right)\right)$.

On the other hand, the inclusion of the fiber in the total space of the fibration $F\left(1^{k-1}, m\right) \rightarrow F\left(1^{k}, m\right) \rightarrow \mathbb{R} P^{m+k-1}$ is surjective in mod 2 cohomology. Therefore the corresponding $\mathbb{F}_{2}$-Serre spectral sequence has trivial coefficients and collapses from its second stage (cf. Theorem 4.4 in page 126 of [20, Part I]). An easy inductive argument ${ }^{3}$ then shows that the $\mathbb{F}_{2}$-Poincaré polynomial of $F\left(1^{k}, m\right)$ is

$$
P(x)=\prod_{i=1}^{k} \frac{1-x^{i+m}}{1-x} .
$$

The proof is complete since $P(1)=\prod_{i=1}^{k}(m+i)$, which is the number of monomials in (4).

\footnotetext{
${ }^{3}$ Alternatively see $[\mathbf{1 4}$, Corollary 9.5.15].
} 
The relations in (3) are a distilled form of a more general (equivalent but nonminimal) set of relations: our proof gives in fact

$$
h_{m+i}\left(x_{1}, \ldots, x_{k-j}\right)=0 \quad \text { if } i>j \geqslant 0 .
$$

(Alternatively, (8) is a consequence of (3) and the obvious inclusions $F\left(1^{k}, m\right) \hookrightarrow$ $\left.F\left(1^{k}, m+1\right) \hookrightarrow F\left(1^{k}, m+2\right) \hookrightarrow \cdots.\right)$ In addition, the obvious action of the symmetric group $\Sigma_{k}$ on (the cohomology of) $F\left(1^{k}, m\right)$ implies that the relations in (8) extend to

$$
h_{m+i}\left(x_{\ell_{1}}, \ldots, x_{\ell_{k-j}}\right)=0,
$$

for any $1 \leqslant \ell_{1}<\cdots<\ell_{k-j} \leqslant k$ with $0 \leqslant j<i \leqslant k$. For instance,

$$
x_{i}^{m+k}=0 \neq x_{i}^{m+k-1} \text { for any } i=1, \ldots, k,
$$

where the non-triviality of $x_{i}^{m+k-1}$ comes from (4). As noted in [21, Example 3.1], this recovers the calculation in $[\mathbf{1 7}]$ of the heights of the generators $x_{i}$ 's. Proposition 2.1 also allows us to recover the calculation of $\operatorname{cat}\left(F\left(1^{k}, m\right)\right.$ ) in $[\mathbf{1 7}]$ (we use the normalized version of the Lusternik-Schnirelmann category, so that a contractible space $X$ has $\operatorname{cat}(X)=0)$ :

Corollary 2.3. $\operatorname{cat}\left(F\left(1^{k}, m\right)\right)=\operatorname{dim}\left(F\left(1^{k}, m\right)\right)=k m+k(k-1) / 2$.

Proof. It is well known that $k m+k(k-1) / 2=\operatorname{dim}\left(F\left(1^{k}, m\right)\right) \geqslant \operatorname{cat}\left(F\left(1^{k}, m\right)\right)$. The latter term is bounded from below by the $\mathbb{F}_{2}$-cup-length of $F\left(1^{k}, m\right)$ which, in view of Proposition 2.1, is no less than $k m+k(k-1) / 2$ since $x_{1}^{m+k-1} x_{2}^{m+k-2} \cdots x_{k}^{m} \neq 0$.

Corollary 2.4. The annihilator of the (non-trivial) class

$$
x_{1}^{m+k-1} x_{2}^{m+k-2} \cdots x_{k}^{m} \in H^{*}\left(F\left(1^{k}, m\right)\right)
$$

is the maximal ideal $H^{>0}\left(F\left(1^{k}, m\right)\right)$ of positive-degree elements. More precisely,

$$
x_{1}^{m+k-1} x_{2}^{m+k-2} \cdots x_{j}^{m+k-j} x_{j}=0,
$$

for $1 \leqslant j \leqslant k$.

Proof. Apply, inductively on $j$, the relation (3) with $i=k-j+1$.

More important for our later purposes is the fact that, as indicated in the proof of Proposition 2.1, the extended relations in (8) can be used in an inductive way to write any polynomial in the $x_{i}$ 's in terms of the basis (4). We next show that the resulting process can be written down with a nice closed formula if certain basis elements are to be neglected.

Proposition 2.5. Let $0 \leqslant j \leqslant i \leqslant k$ with $i \geqslant 1$. In terms of the basis (4), all basis elements $x\left(n_{1}, \ldots, n_{k}\right)$ appearing in the expression of

$$
x_{i}^{m+k-j}+x_{i}^{m+k-i} e_{i-j}\left(x_{1}, \ldots x_{i-1}\right) \in H^{*}\left(F\left(1^{k}, m\right)\right)
$$

have $n_{i}<m+k-i$ and $n_{\ell}=0$ for $\ell>i$.

Proof. The cases $j=0$ and $j=i$ hold vacuously true in view of (9). The case $j=i-1$ follows by observing that a repeated use of (7) allows us to write the relations in (3) 
as

$$
x_{i}^{m+k-i+1}=x_{i}^{m+k-i} h_{1}+x_{i}^{m+k-i-1} h_{2}+\cdots+h_{m+k-i+1},
$$

where the complete symmetric polynomials are evaluated at the variables $x_{1}, \ldots, x_{i-1}$. All other cases $(0<j<i-1)$ follow from an obvious (decreasing) inductive calculation using (6) and the corresponding analogue of (10).

\section{3. $\quad \mathbb{F}_{2}$-zcl bounds for $\mathrm{TC}\left(F\left(1^{k}, m\right)\right)$}

Most of the existing methods to estimate the topological complexity of a given space are cohomological in nature and are based on some form of obstruction theory. One of the most successful methods to estimate Farber's topological complexity is:

Proposition 3.1. Let $X$ have the homotopy type of an $(e-1)$-connected $C W$ complex of dimension d. Then $\operatorname{zcl}_{R}(X) \leqslant \mathrm{TC}(X) \leqslant \frac{2 d}{e}$.

For a proof see [7, Theorems 4 and 7]. Here $R$ is a commutative ring with unit and, if $\Delta: X \hookrightarrow X \times X$ denotes the diagonal inclusion, then $\operatorname{zcl}_{R}(X)$ stands for the maximal number of elements in $\operatorname{ker}\left(\Delta^{*}: H^{*}(X \times X ; R) \rightarrow H^{*}(X ; R)\right)$ having a nontrivial product. ${ }^{4}$ We will only be concerned with $R=\mathbb{Z}_{2}$, and will omit reference of these coefficients while writing cohomology groups. Thus, $\Delta^{*}: H^{*}(X) \otimes H^{*}(X) \rightarrow$ $H^{*}(X)$ is given by cup-multiplication, which explains the notation "zcl" (zero-divisors cup-length) for elements in the kernel of $\Delta^{*}$.

We use the notation $\lambda_{i}$ (resp. $\rho_{i}$ ) for the generators $x_{i}$ on the left (resp. right) tensor factor of $H^{*}\left(F\left(1^{k}, m\right) \times F\left(1^{k}, m\right)\right)=H^{*}\left(F\left(1^{k}, m\right)\right) \otimes H^{*}\left(F\left(1^{k}, m\right)\right)$. The sum $\lambda_{i}+\rho_{i}$, which is a zero-divisor, will be denoted by $z_{i}$.

Lemma 3.2. In the ring $H^{*}\left(F\left(1^{k}, 2^{e}\right)\right)^{\otimes 2}$ we have

$$
\left(z_{1} \cdots z_{k}\right)^{2^{e+1}-1} \neq 0 .
$$

Remark 3.3. When $k \leqslant 2^{e},(11)$ is sharp in the sense that $z_{j}^{2^{e+1}}=\lambda_{j}^{2^{e+1}}+\rho_{j}^{2^{e+1}}=0$ for $1 \leqslant j \leqslant k$, in view of (9). However, such an optimality in (11) is far from holding when $k>2^{e}$. For instance, (11) asserts that $z_{1} z_{2} z_{3} \neq 0$ in $H^{*}\left(F\left(1^{4}\right)\right)^{\otimes 2}$, but we will show in fact (Proposition 3.5 below) that $z_{1}^{3} z_{2}^{3} z_{3}^{2} \neq 0$ in $H^{*}\left(F\left(1^{4}\right)\right)^{\otimes 2}$. A similar phenomenon holds for $F\left(1^{3}, 2\right)$-replacing the use of Proposition 3.5 by Theorem 3.13 below (with $k=3$ ).

Proof of Lemma 3.2. We proceed by induction on $k$. The case for $k=1$ is elementary and well known-note that $F\left(1,2^{e}\right)$ is the real projective space $\mathbb{R P}^{2}$. Assume the result is valid for $k$ and consider the fibration

$$
F\left(1^{k}, 2^{e}\right) \stackrel{\iota}{\rightarrow} F\left(1^{k+1}, 2^{e}\right) \stackrel{\pi}{\rightarrow} F\left(1,2^{e}+k\right)=\mathbb{R} \mathrm{P}^{2^{e}+k},
$$

where $\pi\left(L_{1}, \ldots, L_{k+1}, V\right)=\left(L_{1}, V \oplus \bigoplus_{2 \leqslant i \leqslant k+1} L_{i}\right)$. Since $\iota$ is surjective in cohomology, [20, Theorem 4.4] shows that the Serre spectral sequence for the term-wise cartesian square of (12) has a trivial system of coefficients, and collapses from its second term. The result follows since, by the inductive hypothesis, the left-hand side term in (11) is non-zero in the second stage of the spectral sequence.

\footnotetext{
${ }^{4}$ Twisted cohomology coefficients can be used, but the present setting suffices for our goals.
} 
Proposition 3.1, Corollary 2.3, and Lemma 3.2 yield the estimate in Corollary 3.4 below for the topological complexity of manifolds $F\left(1^{k}, m\right)$ admitting an equatorial inclusion $F\left(1^{k}, 2^{e}\right) \hookrightarrow F\left(1^{k}, m\right)$ with $2^{e} \leqslant m$.

Corollary 3.4. Let e denote the integral part of $\log _{2}(m)$. Then

$$
k\left(2^{e+1}-1\right) \leqslant \operatorname{TC}\left(F\left(1^{k}, m\right)\right) \leqslant k(2 m+k-1) .
$$

Note that the smallest gap in (13) is of $k^{2}$ units (for $m=2^{e}$ ). On the other hand, the lower bound in (13) is optimal in general. For instance, the gap of a unit for $\mathrm{TC}\left(F\left(1,2^{e}\right)\right)$ coming from Corollary 3.4 (with $k=1$ ) is resolved by (2). This is of course compatible with the first assertion in Remark 3.3. But for $k>1$ there is room for improvements of the lower bound in (13) by a zero-divisors cup-length analysis of an intermediate space $F\left(1^{k}, m^{\prime}\right)$ with $2^{e}<m^{\prime} \leqslant m$ and $m^{\prime}$ not a power of 2 . For instance, since (9) yields $z_{j}^{2^{e+1}}=0$ in $H^{*}\left(F\left(1^{2}, 2^{e+1}-2\right)\right)^{\otimes 2}$, the only possibility to improve the lower bound in (13) for $k=2$ via zero-divisors cup-length considerations can come only through the analysis of the case for $F\left(1^{2}, 2^{e+1}-1\right)$. In fact, we next give zero-divisors cup-length bounds for $\mathrm{TC}\left(F\left(1^{k}, m\right)\right)$ inherent to the case $m=$ $2^{e}-1$. As observed at the end of Remark 3.6, our argument will apply only for $k \geqslant 2$-after all, the case of $F\left(1,2^{e}-1\right)$, the real projective space of dimension $2^{e}-1$, has been one of the most difficult situations studied over the years (see, for instance, $[5,15])$.

Proposition 3.5. Assume $k \geqslant 2$ and $e \geqslant 1$. In $H^{*}\left(F\left(1^{k}, 2^{e}-1\right)\right)^{\otimes 2}$ we have

$$
\left(z_{1} \cdots z_{k-1}\right)^{2^{e+1}-1} z_{k}^{2^{e+1}-2} \neq 0 .
$$

Remark 3.6. When $k \leqslant 2^{e}+1$, (14) is almost sharp in the sense that $z_{j}^{2^{e+1}}=0$ for $1 \leqslant j \leqslant k$, in view of $(9)$. Computer calculations show that such an optimality feature fails in general if $k>2^{e}+1$, for instance, with complete Flag manifolds $F\left(1^{k}, 1\right)$. Also worth noticing is that, in Remark 3.7 below, we give evidence suggesting

$$
\left(z_{1} \cdots z_{k}\right)^{2^{e+1}-1}=0
$$

in Proposition 3.5. On the other hand, note that (14) certainly fails for $k=1$.

Proof of Proposition 3.5. The inductive argument in the proof of Lemma 3.2, now replacing (12) by the fibrations

$$
F\left(1^{k}, 2^{e}-1\right) \rightarrow F\left(1^{k+1}, 2^{e}-1\right) \rightarrow F\left(1,2^{e}+k-1\right)=\mathbb{R} P^{2^{e}+k-1},
$$

shows that the general case in Proposition 3.5 follows inductively from the case $k=2$. On the other hand, for the latter case, (9) gives in $H^{*}\left(F\left(1,1,2^{e}-1\right)\right)^{\otimes 2}$

$$
\begin{aligned}
z_{1}^{2^{e+1}-1} z_{2}^{2^{e+1}-2} & =\left(\lambda_{1}+\rho_{1}\right)^{2^{e+1}-1}\left(\lambda_{2}+\rho_{2}\right)^{2^{e+1}-2} \\
& =\left(\lambda_{1}^{2^{e}} \rho_{1}^{2^{e}-1}+\lambda_{1}^{2^{e}-1} \rho_{1}^{2^{e}}\right)\left(\lambda_{2}^{2}+\rho_{2}^{2}\right)^{2^{e}-1} \\
& =\left(\lambda_{1}^{2^{e}} \rho_{1}^{2^{e}-1}+\lambda_{1}^{2^{e}-1} \rho_{1}^{2^{e}}\right)\left(\left(\lambda_{2}^{2}\right)^{2^{e-1}}\left(\rho_{2}^{2}\right)^{2^{e-1}-1}+\left(\lambda_{2}^{2}\right)^{e^{e-1}-1}\left(\rho_{2}^{2}\right)^{2^{e-1}}\right) \\
& =\left(\lambda_{1}^{2^{e}} \rho_{1}^{2^{e}-1}+\lambda_{1}^{2^{e}-1} \rho_{1}^{2^{e}}\right)\left(\lambda_{2}^{2^{e}} \rho_{2}^{2^{e}-2}+\lambda_{2}^{2^{e}-2} \rho_{2}^{2^{e}}\right) .
\end{aligned}
$$

Further, if $\mu$ stands for either $\lambda$ or $\rho$, the relations (3) give $\mu_{2}^{2^{e}}=\mu_{1}^{2} A_{\mu}+\mu_{1} \mu_{2}^{2^{e}-1}$. 
Thus,

$$
\begin{aligned}
& z_{1}^{2^{e+1}}-1 z_{2}^{2^{e+1}-2} \\
& =\left(\lambda_{1}^{2^{e}} \rho_{1}^{2^{e}-1}+\lambda_{1}^{2^{e}-1} \rho_{1}^{2^{e}}\right)\left(\lambda_{2}^{2^{e}} \rho_{2}^{2^{e}-2}+\lambda_{2}^{2^{e}-2} \rho_{2}^{2^{e}}\right) \\
& =\left(\lambda_{1}^{2^{e}} \rho_{1}^{2^{e}-1}+\lambda_{1}^{2^{e}-1} \rho_{1}^{2^{e}}\right)\left(\left(\lambda_{1}^{2} A_{\lambda}+\lambda_{1} \lambda_{2}^{2^{e}-1}\right) \rho_{2}^{2^{e}-2}+\lambda_{2}^{2^{e}-2}\left(\rho_{1}^{2} A_{\rho}+\rho_{1} \rho_{2}^{2^{e}-1}\right)\right) \\
& \quad=\lambda_{1}^{2^{e}} \rho_{1}^{2^{e}-1} \cdot \lambda_{2}^{2^{e}-2} \rho_{1} \rho_{2}^{2^{e}-1}+\lambda_{1}^{2^{e}-1} \rho_{1}^{2^{e}} \cdot \lambda_{1} \lambda_{2}^{2^{e}-1} \rho_{2}^{2^{e}-2} \\
& \quad=\lambda_{1}^{2^{e}} \lambda_{2}^{2^{e}-2} \rho_{1}^{2^{e}} \rho_{2}^{2^{e}-1}+\lambda_{1}^{2^{e}} \lambda_{2}^{2^{e}-1} \rho_{1}^{2^{e}} \rho_{2}^{2^{e}-2} .
\end{aligned}
$$

The result follows as the two monomials in the last expression are basis elements.

Remark 3.7. Before discussing the implications of Proposition 3.5 to the topological complexity of flag manifolds, we make a brief pause to say a few words about the sharpness of Proposition 3.5 when $2 \leqslant k \leqslant 2^{e}+1$-hypothesis that will be in force in this paragraph. Since $0=z_{i}^{2^{e+1}} \in H^{*}\left(F\left(1^{k}, 2^{e}-1\right)\right)^{\otimes 2}$ for all $i$, the triviality of any product $z_{i_{1}} \cdots z_{i_{t}}$ with $t \geqslant\left(2^{e+1}-1\right) k$ is equivalent to

$$
\left(z_{1} \cdots z_{k}\right)^{2^{e+1}-1}=0 .
$$

Proving (15) presents a major challenge (not addressed in this work). Checking the validity of (15) for $k=2$ is easy in view of the last expression for $z_{1}^{2^{e+1}-1} z_{2}^{2^{e+1}-2}$ at the end of the proof of Proposition 3.5. We have checked the validity of (15) for $k \in\{3,4\}$ with the help of a computer, but the task quickly becomes computationally prohibitive for larger values of $k$ as the number of basis elements in the expression on the left-hand term of (14) increases extremely fast as $k$ grows: 16 basis elements are need for $k=3$, while the number of required basis elements increases to 1128 for $k=4$ - the sum of which would have to vanish after multiplying by $z_{k}$, should (15) be true.

Corollary 3.8. Let $e$ denote the integral part of $\log _{2}(m+1)$. If $e \geqslant 1$ and $k \geqslant 2$, then

$$
k\left(2^{e+1}-1\right)-1 \leqslant \operatorname{TC}\left(F\left(1^{k}, m\right)\right) \leqslant k(2 m+k-1) .
$$

Remark 3.9. The smallest gap in (16) is of $k(k-2)+1$ units (for $m=2^{e}-1$ ). The case of $F\left(1^{2}, 2^{e}-1\right)$ (e.g., the closed parallelizable 3 -manifold $\left.F\left(1^{3}\right)\right)$ is particularly appealing, as the corresponding gap is only of one unit.

Example 3.10. It is elementary to see that, when $k=1$, Corollary 3.4 captures all the TC-information cohomologically available from Lemma 3.2. Indeed, the relations $z_{1}^{2^{e+1}-1} \neq 0=z_{1}^{2^{e+1}}$, holding in $H^{*}\left(F\left(1,2^{e}\right)\right)^{\otimes 2}$, clearly hold in any $H^{*}(F(1, m))^{\otimes 2}$ with $2^{e} \leqslant m<2^{e+1}$. Similarly, Corollaries 3.4 and 3.8 capture all the TC-information available from Lemma 3.2 and Proposition 3.5 if $k=2$. For $z_{1}^{2^{e+1}-1} z_{2}^{2^{e+1}-2} \neq 0$ holds sharply in $H^{*}\left(F\left(1,1,2^{e}-1\right)\right)^{\otimes 2}$ (cf. Remark 3.7), and $z_{1}^{2^{e+1}-1} z_{2}^{2^{e+1}-1} \neq 0$ holds sharply in $H^{*}(F(1,1, m))^{\otimes 2}$ for $2^{e} \leqslant m \leqslant 2^{e+1}-2$ (cf. Remark 3.3).

Example 3.11 below (and extensive computer calculations) seem to suggest that all the zcl-information for $F\left(1^{k}, m\right)$ is contained in the cases $m=2^{e}-\delta$ with $0 \leqslant \delta<k$. The analysis of the corresponding zcl properties is the subject of the remainder of this section (see Theorem 3.13). 
Example 3.11. Apply the inductive argument in the proofs of Lemma 3.2 and Proposition 3.5 to the fibration $F\left(1,2^{e}-2\right) \rightarrow F\left(1^{k}, 2^{e}-2\right) \rightarrow F\left(1^{k-1}, 2^{e}-1\right)$. For $k \geqslant 3$ and $e \geqslant 1$, Proposition 3.5 gives $\left(z_{1} \cdots z_{k-2}\right)^{2^{e+1}-1} z_{k-1}^{2^{e+1}-2} \neq 0$ in $H^{*}\left(F\left(1^{k-1}, 2^{e}-\right.\right.$ $1))^{\otimes 2}$. Since $0 \neq z_{1}^{2^{e}-1} \in H^{*}\left(F\left(1,2^{e}-2\right)\right)^{\otimes 2}$ is a standard calculation for $e \geqslant 2$, we see

$$
\left(z_{1} \cdots z_{k-2}\right)^{2^{e+1}-1} z_{k-1}^{2^{e+1}-2} z_{k}^{2^{e}-1} \neq 0 \text { in } H^{*}\left(F\left(1^{k}, 2^{e}-2\right)\right)^{\otimes 2} \text { if } k \geqslant 3 \text { and } e \geqslant 2 .
$$

What is remarkable in (17) is that, although this argument is really measuring the zero-divisors cup-length of a graded object associated to $H^{*}\left(F\left(1^{k}, 2^{e}-2\right)\right)^{\otimes 2}$, extensive computer calculations suggest that no cohomological information has been missed.

Remark 3.12. At a first glance, (2) and Remark 3.9 might suggest that the methods in this paper could lead to estimate the TC of $F\left(1^{3}, 2^{e}-2\right)$ with an error of at most a unit. The error, however, increases exponentially with $e$ (see Example 3.15). Yet, as shown in Section 4 below, the corresponding $\mathrm{TC}_{s}$ estimates for $s \geqslant 3$ will in fact be sharp.

The argument leading to (17) can be iterated with the fibrations

$$
F\left(1,2^{e}-\delta\right) \rightarrow F\left(1^{k}, 2^{e}-\delta\right) \rightarrow F\left(1^{k-1}, 2^{e}-\delta+1\right),
$$

for $\delta \geqslant 2$ (but note that the case $\delta=1$ fails to recover Proposition 3.5) to get the following generalizations of Lemma 3.2 and Proposition 3.5, and of Corollaries 3.4 and 3.8:

Theorem 3.13. The following assertions hold in $H^{*}\left(F\left(1^{k}, m\right)\right)^{\otimes 2}$ :

(a) For $m+k \leqslant 2^{e+1}$ and $1 \leqslant i \leqslant k, z_{i}^{2^{e+1}}=0$.

(b) For $m=2^{e}-\delta$ with $k>\delta \geqslant 0$ and $2^{e-1} \geqslant \delta$,

$$
\left(z_{1} \cdots z_{k-\delta}\right)^{2^{e+1}-1} z_{k-\delta+1}^{2^{e+1}-2}\left(z_{k-\delta+2} \cdots z_{k}\right)^{2^{e}-1} \neq 0 .
$$

Corollary 3.14. Let $k$ and $m$ be positive integers, $\delta \in\{0,1, \ldots, k-1\}$, and set $\epsilon=$ $\min (\delta, 1)$ and $\alpha(r)=\max (0, r)$. If a nonnegative integer e satisfies $2 \delta \leqslant 2^{e} \leqslant m+\delta$, then

$$
(k-\delta+\epsilon)\left(2^{e+1}-1\right)+\alpha\left((\delta-1)\left(2^{e}-1\right)\right)-\epsilon \leqslant \operatorname{TC}\left(F\left(1^{k}, m\right)\right) \leqslant k(2 m+k-1) .
$$

Due to the form of the exponents of the factors on the left-hand side of (18), the gap in (19) becomes in general larger as the parameter $\delta$ increases. Still, as shown in the following examples, there are concrete situations where Corollary 3.14 yields better lower bounds for larger values of $\delta$.

Example 3.15. Obtaining the sharpest information from Theorem 3.13 and Corollary 3.14 for a fixed flag manifold $F\left(1^{k}, m_{0}\right)$ usually requires choosing a suitable combination of parameters $(e, \delta)$ with $2^{e}-\delta \leqslant m_{0}$ (so that the non-triviality of a cohomology class in $F\left(1^{k}, m_{0}\right)$ can be obtained, via Theorem 3.13, from the nontriviality of its restriction to $\left.F\left(1^{k}, 2^{e}-\delta\right)\right)$. Take, for instance, the case of $F\left(1^{3}, 2\right)$ where the conclusion of item (b) in Theorem 3.13 with $\delta=0$ is $\left(z_{1} z_{2} z_{3}\right)^{3} \neq 0$, but 
the conclusion with $\delta=2$ is in fact $z_{1}^{7} z_{2}^{6} z_{3}^{3} \neq 0$. Alternatively, the case $\delta=0$ in Corollary 3.14 implies $\operatorname{TC}\left(F\left(1^{3}, 6\right)\right) \geqslant 21$, while the case $\delta=2$ yields the stronger estimate $\mathrm{TC}\left(F\left(1^{3}, 6\right)\right) \geqslant 36$. In particular, the smallest gap in (19) for $F\left(1^{3}, 6\right)$ is of 6 units and corresponds to $\delta=2$. More generally, for $e \geqslant 2$, the smallest gap in (19) for $F\left(1^{3}, 2^{e}-2\right)$ is of $2^{e}-2$ units. In particular, $\operatorname{TC}\left(F\left(1^{3}, 2\right)\right) \in\{16,17,18\}$ - a gap of only two units.

As indicated in Remarks 3.3 and 3.6, the lower bound in (19) tends to get weaker as $k$ is larger than $2^{e}-\delta$. Extreme cases hold (with $\left.(e, \delta) \in\{(0,0),(1,1)\}\right)$ for complete flag manifolds $F\left(1^{k}, 1\right)$.

\section{Higher topological complexity}

We have seen that the methods in this paper give almost-sharp estimates for the topological complexity of flag manifolds $F\left(1,2^{e}\right)$ and $F\left(1^{2}, 2^{e}-1\right)$. This section's goal is to show that, in the realm of higher topological complexity, the cohomological estimates become sharp and, above all, valid for other flag manifolds of the form $F\left(1^{k}, 2^{e}-k+1\right)$. In general, our results show that, as $s$ increases, the cohomological method becomes better suited to estimate $\mathrm{TC}_{s}\left(F\left(1^{k}, m\right)\right)$. This point will be made precise in Remark 4.2 and Corollary 4.8 below.

Proposition 4.1 below gives the $\mathrm{TC}_{s}$-analogue of Proposition 3.1. Here $\mathrm{zcl}_{s, R}(X)$, the $s$-th zero-divisors cup-length of $X$, stands for the maximal number of elements in $\operatorname{ker}\left(\Delta_{s}^{*}: H^{*}\left(X^{s} ; R\right) \rightarrow H^{*}(X ; R)\right)$ having a non-trivial product, and $\Delta_{s}: X \rightarrow X^{s}$ is the iterated diagonal.

Proposition 4.1 ([22, Proposition 3.4]). Let $X$ have the homotopy type of an $(e-1)$-connected $C W$ complex of dimension $d$. Then

$$
\operatorname{zcl}_{s, R}(X) \leqslant \operatorname{TC}_{s}(X) \leqslant \frac{s d}{e} .
$$

Remark 4.2. Let $G(k, m, s)$ denote the gap in (20) for $R=\mathbb{Z}_{2}$ and $X=F\left(1^{k}, m\right)$, i.e., $G(k, m, s)=s d_{k, m}-\mathrm{zcl}_{s, \mathbb{Z}_{2}}\left(F\left(1^{k}, m\right)\right)$ where $d_{k, m}=k m+k(k-1) / 2$ (cf. Corollary 2.3). Corollary 4.8 below indicates that, for $k$ and $m$ fixed, the sequence of nonnegative integers $\{G(k, m, s)\}_{s \geqslant 2}$ is monotonically decreasing and, therefore, eventually constant. In fact, in the main result of this section (Theorem 4.3 below), the monotonic phenomenon holds with a zero limiting value, $\lim _{s \mapsto \infty} G(k, m, s)=0$, thus getting sharp results in this particular case.

Theorem 4.3. For positive integers $e, k$ and $s$ with $e \geqslant 1+\left\lfloor\frac{k-1}{2}\right\rfloor$ and $k \leqslant 3 \leqslant s$, $\mathrm{zcl}_{s, \mathbb{Z}_{2}}\left(F\left(1^{k}, 2^{e}-k+1\right)\right)=\mathrm{TC}_{s}\left(F\left(1^{k}, 2^{e}-k+1\right)\right)=s \operatorname{dim}\left(F\left(1^{k}, 2^{e}-k+1\right)\right)$.

Theorem 4.3 is an immediate consequence of Proposition 4.1 and the inequality $s \operatorname{dim}\left(F\left(1^{k}, 2^{e}-k+1\right)\right) \leqslant \mathrm{zcl}_{s, \mathbb{Z}_{2}}\left(F\left(1^{k}, 2^{e}-k+1\right)\right)$, which will be established in Propositions 4.4 and 4.5 for $k \leqslant 3 \leqslant s$ by identifying non-trivial products with suitably many $s$-th zero-divisors as factors.

For $1 \leqslant i \leqslant s$ and $1 \leqslant j \leqslant k$, let $x_{i, j}$ be the pullback class $\pi_{i}^{*}\left(x_{j}\right) \in H^{*}\left(F\left(1^{k}, m\right)\right)^{\otimes s}$ where $\pi_{i}: F\left(1^{k}, m\right)^{s} \rightarrow F\left(1^{k}, m\right)$ is the $i$-th projection $(1 \leqslant i \leqslant s$ and $1 \leqslant j \leqslant k)$, and let $z_{i, j}$ stand for the $s$-th zero-divisor $x_{1, j}+x_{i, j}$. We will deal with the basis (4) and 
its tensor product basis

$$
\prod_{i=1}^{s} x_{i}\left(n_{i, 1}, \ldots n_{i, k}\right), \quad 0 \leqslant n_{i, j} \leqslant m+k-j,
$$

where $x_{i}\left(n_{i, 1}, \ldots n_{i, k}\right)=\pi_{i}^{*}\left(x\left(n_{i, 1}, \ldots n_{i, k}\right)\right)$.

Proposition 4.4. For $s \geqslant 3$ and $e \geqslant 1$,

1. $z_{2,1}^{2^{e+1}-1} z_{3,1}^{2^{e}+1} z_{4,1}^{2^{e}} \cdots z_{s, 1}^{2^{e}}$ is non-trivial in the cohomology ring $H^{*}\left(F\left(1,2^{e}\right)\right)^{\otimes s}$.

2. $\left(z_{2,1}^{2^{e+1}-1} z_{2,2}^{2^{e+1}-2}\right) \cdot\left(z_{3,1}^{2^{e}-1} z_{3,2}^{2^{e}+1}\right) \cdot\left(z_{4,1}^{2^{e}} z_{4,2}^{2^{e}-1}\right) \cdots\left(z_{s, 1}^{2^{e}} z_{s, 2}^{2^{e}-1}\right)$ is non-trivial in the cohomology ring $H^{*}\left(F\left(1^{2}, 2^{e}-1\right)\right)^{\otimes s}$.

Proposition 4.5. For $s \geqslant 3$ and $e \geqslant 2$,

$$
0 \neq\left(z_{2,1}^{2^{e+1}-1} z_{2,2}^{2^{e+1}-2} z_{2,3}^{2^{e}-1}\right) \cdot\left(z_{3,1}^{2^{e}-1} z_{3,2}^{2^{e}-1} z_{3,3}^{2^{e+1}-3}\right) \cdot \prod_{i=4}^{s}\left(z_{i, 1}^{2^{e}} z_{i, 2}^{2^{e}-1} z_{i, 3}^{2^{e}-2}\right)
$$

in the cohomology ring $H^{*}\left(F\left(1^{3}, 2^{e}-2\right)\right)^{\otimes s}$.

Remark 4.6. Note that the powers of the factors $z_{2, j}$ in the three products above coincide with the relevant power(s) of the products in (11) with $k=1$, (14) with $k=2$, and $(17)$ for $k=3$. In the present case $(s \geqslant 3)$, the form of the powers of the factors $z_{3, j}$ is what allows us to get sharp results. Also worth mentioning is the possibility that Theorem 4.3 could hold true by relaxing the restriction " $k \leqslant 3 \leqslant s$ " to " $k \leqslant s$ " (see Remark 4.14 for a more general possibility). The proof of such an assertion seems to require computational input (suggesting suitable generalizations of Propositions 4.4 and 4.5) that does not seem to be currently available with today's computer capabilities.

Lemma 4.7 below implies that it suffices to prove Propositions 4.4 and 4.5 for $s=3$.

Lemma 4.7. For $2 \leqslant i \leqslant s$, the expression in $H^{*}\left(F\left(1^{k}, m\right)\right)^{\otimes s}$ of

$$
z_{i, 1}^{m+k-1} z_{i, 2}^{m+k-2} \cdots z_{i, k-1}^{m+1} z_{i, k}^{m}+x_{i}(m+k-1, m+k-2, \cdots, m+1, m)
$$

in terms of the basis in (21) involves only basis elements of the form

$$
x_{1}\left(r_{1}, \ldots, r_{k}\right) \cdot x_{i}\left(t_{1}, \ldots, t_{k}\right),
$$

with $r_{j}>0$ for some $j \in\{1, \ldots, k\}$ (so $t_{j^{\prime}}<m+k-j^{\prime}$ for some $j^{\prime} \in\{1, \ldots, k\}$ ).

Proof. Expand out $z_{i, 1}^{m+k-1} z_{i, 2}^{m+k-2} \cdots z_{i, k-1}^{m+1} z_{i, k}^{m}$ and notice that all the resulting monomials are basis elements.

Corollary 4.8. For $k, m \geqslant 1, G(k, m, 2) \geqslant G(k, m, 3) \geqslant G(k, m, 4) \geqslant \cdots \geqslant 0$.

Proof. If $z \in H^{*}\left(F\left(1^{k}, m\right)\right)^{\otimes s}$ is some non-trivial product of $s$-th zero-divisors, then Lemma 4.7 implies that $z \cdot z_{s+1,1}^{m+k-1} z_{s+1,2}^{m+k-2} \cdots z_{s+1, k-1}^{m+1} z_{s+1, k}^{m} \in H^{*}\left(F\left(1^{k}, m\right)\right)^{\otimes(s+1)}$ is non-trivial too. The result then follows from the bare definition of the function $G(k, m, s)$. 
Propositions 4.4 and 4.5 are proved by direct computation of the given products. In all cases, advantage is taken of the fact that the products lie in the top dimension $s\left(k m+\left(\begin{array}{c}k \\ 2\end{array}\right)\right)$ of the relevant ring $H^{*}\left(F\left(1^{k}, m\right)\right)^{\otimes s}$, where the additive basis $(21)$ reduces to the single element

$$
\prod_{i=1}^{s} x_{i}(m+k-1, m+k-2, \ldots, m+1, m) .
$$

Explicitly, we use the inductive process indicated in the proof of Proposition 2.1, except that, since (22) is the only basis element we care about, the extended relations in $(8)$ can be replaced by the relations

$$
x_{i}^{m+k-j}=x_{i}^{m+k-i} e_{i-j}\left(x_{1}, \ldots x_{i-1}\right), \text { for } 0 \leqslant j \leqslant i \leqslant k \text { and } i \geqslant 1,
$$

coming from Proposition 2.5. Proof details for Proposition 4.4 are similar (and easier) than those for Proposition 4.5, so we only focus on the latter case.

Proof of Proposition 4.5. By Lemma 4.7 (see also the proof of Corollary 4.8), we only need to consider the case $s=3$. We will show that for $e \geqslant 2$,

$$
\left(z_{2,1}^{2^{e+1}-1} z_{2,2}^{2^{e+1}-2} z_{2,3}^{2^{e}-1}\right) \cdot\left(z_{3,1}^{2^{e}-1} z_{3,2}^{2^{e}-1} z_{3,3}^{2^{e+1}-3}\right)=\prod_{i=1}^{3} x_{i, 1}^{2^{e}} x_{i, 2}^{2^{e}-1} x_{i, 3}^{2^{e}-2},
$$

the top basis element in $H^{*}\left(F\left(1^{3}, 2^{e}-2\right)\right)^{\otimes 3}$

The mod-2 arithmetic of binomial coefficients, and the fact that $x_{i, j}^{2^{e}+1}=0$ give

$$
z_{3,3}^{2^{e+1}-3}=\left(x_{1,3}+x_{3,3}\right)^{2^{e+1}-3}=x_{1,3}^{2^{e}} x_{3,3}^{2^{e}-3}+x_{1,3}^{2^{e}-3} x_{3,3}^{2^{e}}
$$

(of course, this uses the hypothesis $e \geqslant 2$ ). Due to the form of the relations (23) - or (8) for that matter - and since $x_{3,3}^{2^{e}-3}$ is a basis element, the term $x_{1,3}^{2^{e}} x_{3,3}^{2^{e}-3}$ above cannot contribute to the top basis element. In other words, the considerations around (22) imply that the product of the term $x_{1,3}^{2^{e}} x_{3,3}^{2^{e}}-3$ with the first five powers on the left of (24) vanishes. Such an argument will be used repeatedly in what follows, and will simply be referred to by using a "三” symbol. In these terms, the relations (23) allow us to extend (25) to

$$
\begin{aligned}
z_{3,3}^{2^{e+1}-3}=x_{1,3}^{2^{e}} x_{3,3}^{2^{e}-3} & +x_{1,3}^{2^{e}-3} x_{3,3}^{2^{e}} \equiv x_{1,3}^{2^{e}-3} x_{3,3}^{2^{e}} \equiv x_{1,3}^{2^{e}-3} x_{3,1} x_{3,2} \cdot x_{3,3}^{2^{e}-2}, \\
z_{3,2}^{2^{e}-1} z_{3,3}^{2^{e+1}-3} & \equiv z_{3,2}^{2^{e}-1} x_{1,3}^{2^{e}-3} x_{3,1} x_{3,2} \cdot x_{3,3}^{2^{e}-2} \\
& =\left(x_{1,2}+x_{3,2}\right)^{2^{e}-1} x_{1,3}^{2^{e}-3} x_{3,1} x_{3,2} \cdot x_{3,3}^{2^{e}-2} \\
& \equiv\left(x_{1,2} x_{3,2}^{2^{e}-2}+x_{3,2}^{2^{e}-1}\right) x_{1,3}^{2^{e}-3} x_{3,1} x_{3,2} \cdot x_{3,3}^{2^{e}-2} \\
& =x_{1,3}^{2^{e}-3} x_{3,1}\left(x_{1,2}+x_{3,1}\right) \cdot x_{3,2}^{2^{e}-1} x_{3,3}^{2^{e}-2},
\end{aligned}
$$

and

$$
\begin{aligned}
z_{3,1}^{2^{e}-1} z_{3,2}^{2^{e}-1} z_{3,3}^{2^{e+1}-3} & \equiv\left(x_{1,1}+x_{3,1}\right)^{2^{e}-1} x_{1,3}^{2^{e}-3} x_{3,1}\left(x_{1,2}+x_{3,1}\right) \cdot x_{3,2}^{2^{e}-1} x_{3,3}^{2^{e}-2} \\
& \equiv x_{1,3}^{2^{e}-3}\left(x_{1,1} x_{3,1}^{2^{e}-2}+x_{3,1}^{2^{e}-1}\right) x_{3,1}\left(x_{1,2}+x_{3,1}\right) \cdot x_{3,2}^{2^{e}-1} x_{3,3}^{2^{e}-2} \\
& =x_{1,3}^{2^{e}-3}\left(x_{1,1}+x_{1,2}\right) \cdot x_{3,1}^{2^{e}} x_{3,2}^{2^{e}-1} x_{3,3}^{2^{e}-2}
\end{aligned}
$$

An entirely similar (and straightforward) calculation gives

$$
z_{2,1}^{2^{e+1}-1} z_{2,2}^{2^{e+1}-2} z_{2,3}^{2^{e}-1} \equiv\left(x_{1,1}^{2^{e}} x_{1,2}^{2^{e}-2} x_{1,3}+x_{1,1}^{2^{e}-1} x_{1,2}^{2^{e}}\right) \cdot x_{2,1}^{2^{e}} x_{2,2}^{2^{e}-1} x_{2,3}^{2^{e}-2},
$$


and the result then follows since an additional (and much simpler) such computation gives $x_{1,3}^{2^{e}-3}\left(x_{1,1}+x_{1,2}\right) \cdot\left(x_{1,1}^{2^{e}} x_{1,2}^{2^{e}-2} x_{1,3}+x_{1,1}^{2^{e}-1} x_{1,2}^{2^{e}}\right) \equiv x_{1,1}^{2^{e}} x_{1,2}^{2^{e}-1} x_{1,3}^{2^{e}-2}$.

The Serre spectral sequence method used in Section 3 could now be coupled with Propositions 4.4 and 4.5 to get an extension of Theorem 4.3 on the lines of Corollary 3.14. However, such a task would need to be done in a carefully selective way as, in some cases, the direct computations in the previous proof give better results. In fact, as the following example suggests (see also the proof of Proposition 4.10), best results can be obtained by a suitable combination of both techniques.

Example 4.9. Proposition 4.4(1) and the Serre spectral sequence applied to the fibration $F\left(1,2^{e}\right) \rightarrow F\left(1,1,2^{e}\right) \rightarrow F\left(1,2^{e}+1\right)$ (with $e \geqslant 1$ ) yield the non-triviality of

$$
\left(z_{2,1}^{2^{e+1}-1} z_{2,2}^{2^{e+1}-1}\right) \cdot\left(z_{3,1}^{2^{e}+1} z_{3,2}^{2^{e}+1}\right) \cdot\left(z_{4,1}^{2^{e}} z_{4,2}^{2^{e}}\right) \cdots\left(z_{s, 1}^{2^{e}} z_{s, 2}^{2^{e}}\right) \in H^{*}\left(F\left(1,1,2^{e}\right)\right)^{\otimes s},
$$

for $s \geqslant 3$. But one can do better. For instance, a direct argument (spelled out in Proposition 4.10 below) gives in fact the non-triviality of

$$
\left(z_{2,1}^{3} z_{2,2}^{3}\right) \cdot\left(z_{3,1}^{3} z_{3,2}^{3}\right) \cdot\left(z_{4,1}^{3} z_{4,2}^{2}\right) \cdots\left(z_{s, 1}^{3} z_{s, 2}^{2}\right) \in H^{*}(F(1,1,2))^{\otimes s},
$$

for $s \geqslant 3$, so that $5 s-3 \leqslant \mathrm{TC}_{s}(F(1,1,2)) \leqslant 5 s$. As a result we have that $G(2,2, s) \leqslant$ 3 provided $s \geqslant 3$ (recall from Corollary 3.4 that $G\left(2,2^{e}, 2\right)=4$ ). In fact, extensive computer computations (not given here) suggest that

$$
G(2,2, s)=3 \text { when } s \geqslant 3 .
$$

The key point then comes from the fact that Theorem 4.11 below gives the sharper result ${ }^{5}$

$$
G\left(2,2^{e}, s\right) \leqslant 1 \text { for } s \geqslant 3 \text { and } e \geqslant 2 .
$$

Proposition 4.10. The element in (26) is non-zero.

Proof. The assertion for $s=3$ has been observed in the first sentence of Example 4.9. The case $s \geqslant 4$ then follows from (the proof of) Corollary 4.8.

As anticipated in Example 4.9, we also describe, for $s \geqslant 3$ and $e \geqslant 2$, an almost sharp estimate for $\mathrm{TC}_{s}\left(F\left(1,1,2^{e}\right)\right)$.

Theorem 4.11. For $e \geqslant 2$ and $s \geqslant 3$,

$$
0 \neq\left(z_{2,1}^{2^{e+1}-1} z_{2,2}^{2^{e+1}-1}\right) \cdot\left(z_{3,1}^{2^{e}+1} z_{3,2}^{2^{e}+3}\right) \cdot\left(z_{4,1}^{2^{e}+1} z_{4,2}^{2^{e}}\right) \cdots\left(z_{s, 1}^{2^{e}+1} z_{s, 2}^{2^{e}}\right)
$$

in $H^{*}\left(F\left(1,1,2^{e}\right)\right)^{\otimes s}$, consequently $s\left(2^{e+1}+1\right)-1 \leqslant \mathrm{TC}_{s}\left(F\left(1,1,2^{e}\right)\right) \leqslant s\left(2^{e+1}+1\right)$.

Remark 4.12. Just as observed in Remark 4.6 in the case of Propositions 4.4 and 4.5, the powers of the factors $z_{2, j}$ in the product element of Theorem 4.11 coincide with the relevant powers of the product in (11) for $k=2$. The authors believe that such a phenomenon should shed light on possible generalizations of Theorems 4.3 and 4.11.

Remark 4.13. Theorem 4.11 fails for $e=1$, as $z_{3,2}^{4}=x_{1,2}^{4}+x_{3,2}^{4}$, which vanishes in $H^{*}(F(1,1,2))^{\otimes s}$ in view of $(9)$.

\footnotetext{
${ }^{5}$ Computer calculations suggest that, in fact, $G\left(2,2^{e}, s\right)=1$ for $e \geqslant 2$ and $s \geqslant 3$.
} 
Proof of Theorem 4.11. As in previous proofs, we can safely assume $s=3$. Further, although we should not focus now on the top dimensional basis element (22), the needed verifications are similar to those in the proof of Proposition 4.5. Indeed, this time we indicate how, for $e \geqslant 2$, the basis element $x_{1,1}^{2^{e}} x_{1,2}^{2^{e}} \cdot x_{2,1}^{2^{e}+1} x_{2,2}^{2^{e}} \cdot x_{3,1}^{2^{e}+1} x_{3,2}^{2^{e}}$ appears in the expression of $\left(z_{2,1}^{2^{e+1}-1} z_{2,2}^{2^{e+1}-1}\right) \cdot\left(z_{3,1}^{2^{e}+1} z_{3,2}^{2^{e}+3}\right)$ in terms of the tensor basis (21). The hypothesis $e \geqslant 2$ is used for the analysis of the mod-2 arithmetics of binomial coefficients. That being said, the calculation details can easily be carried out by the diligent reader. As a guide, we note that the three key steps are

$$
\begin{aligned}
z_{3,1}^{2^{e}+1} z_{3,2}^{2^{e}+3} & \equiv x_{1,1} x_{1,2}^{2}+x_{1,2}^{3}, \\
z_{2,1}^{2^{e+1}-1} z_{2,2}^{2^{e+1}-1} & \equiv x_{1,1}^{2^{e}-1} x_{1,2}^{2^{e}-2}+x_{1,1}^{2^{e}-2} x_{1,2}^{2^{e}-1},
\end{aligned}
$$

and the easy fact that $\left(x_{1,1} x_{1,2}^{2}+x_{1,2}^{3}\right) \cdot\left(x_{1,1}^{2^{e}-1} x_{1,2}^{2^{e}-2}+x_{1,1}^{2^{e}-2} x_{1,2}^{2^{e}-1}\right)=x_{1,1}^{2^{e}} x_{1,2}^{2^{e}}$.

Remark 4.14. The results in this section suggest that purely cohomological methods could be used to give, for positive integers $i$ and $k$, an estimate of the higher topological complexity of $F\left(1^{k}, 2^{e}-k+i\right)$ giving $G\left(k, 2^{e}-k+i, s\right)<i$ provided $e$ is sufficiently large. An interesting additional restriction of the form $k+i-1 \leqslant s$, which would be compatible with the corresponding restrictions in Theorems 4.3 and 4.11 (as well as with computer calculations not shown here), might also be needed.

\section{Surfaces}

While Farber's topological complexity of a closed orientable surface was computed in the early stages of the theory [7], the non-orientable case has stood as a particularly intriguing task. For the lowest genus, it is known that $\operatorname{TC}\left(\mathbb{R P}^{2}\right)=\operatorname{Imm}\left(\mathbb{R P}^{2}\right)=3$, whereas a sophisticated obstruction theoretic analysis is given in $[\mathbf{6}]$ to prove that (just as for oriented surfaces of genus at least 2) the topological complexity of a closed non-orientable surface of genus at least 4 is 4 . The topological complexity of the non-orientable surfaces of genus 2 or 3 remain still undecided. The goal of this section is to show that, just as for the semi-complete flag manifolds in the previous section, the higher topological complexity of all (orientable or not) closed surfaces is fully accessible from cohomological methods.

Proposition 5.1. Let $\Sigma$ stand for a closed surface (orientable or not) other than the sphere or the torus. Then $\mathrm{TC}_{s}(\Sigma)=2 s$ for all $s \geqslant 3$.

The 2-sphere $S^{2}$ and the torus $T$ are true exceptional cases, as they have $\operatorname{TC}_{s}\left(S^{2}\right)=$ $s$ and $\mathrm{TC}_{s}(T)=2 s-2$ for any $s \geqslant 2$ (see $\left.[\mathbf{1}]\right)$.

Proof. Proof details for the oriented case are freely accessible from [11], and we only consider here the non-oriented case. For a positive integer $n$, let $N_{n}=\sharp_{i=1}^{n} \mathbb{R} \mathrm{P}^{2}$ denote the closed non-orientable surface of genus $n$. Recall that the mod 2 cohomology ring $H^{*}\left(N_{n}\right)$ is generated by 1 -dimensional classes $a_{1}, \ldots, a_{n}$ subject only to the relations $a_{i}^{2}=a_{j}^{2}$ and $a_{i} a_{j}=0$ for all $1 \leqslant i, j \leqslant n$ with $i \neq j$, together with the relation $a_{1}^{3}=0$ when $n=1$ (the latter relation is obviously superfluous if $n>1$ ). In particular, there is a ring monomorphism $\varphi: H^{*}\left(N_{1}\right) \hookrightarrow H^{*}\left(N_{n}\right)$ determined by $\varphi\left(a_{1}\right)=a_{1}$. The corresponding ring monomorphism $\varphi_{s}:=\varphi^{\otimes s}: H^{*}\left(N_{1}\right)^{\otimes s} \hookrightarrow H^{*}\left(N_{n}\right)^{\otimes s}$ satisfies 
$\varphi_{s}\left(z_{i, 1}\right)=c_{i, 1}$ for $2 \leqslant i \leqslant s$, where the classes $z_{i, 1}$ are defined in the paragraph following Remark 4.2 (note that $N_{1}=\mathbb{R} \mathrm{P}^{2}=F(1,2)$ ), and the $s$-th zero-divisors $c_{i, 1}$ are given by

$$
c_{i, 1}=\underbrace{a_{1} \otimes 1 \otimes \cdots \otimes 1}_{s \text { factors }}+\underbrace{1 \otimes \cdots \otimes 1 \otimes a_{1}^{i} \otimes 1 \otimes \cdots \otimes 1}_{s \text { factors }} \in H^{*}\left(N_{n}\right)^{\otimes s} .
$$

Here an $i$ on top of a tensor factor indicates the coordinate where the factor appears. The equality $\mathrm{TC}_{s}\left(N_{n}\right)=2 s$ (for $s \geqslant 3$ ) now follow from Proposition 4.1 since

$$
c_{2,1}^{3} c_{3,1}^{3} \prod_{i=4}^{s}\left(c_{i, 1}\right)^{2} \neq 0
$$

in view of Proposition 4.4(1).

\section{References}

[1] I. Basabe, J. González, Y.B. Rudyak, and D. Tamaki. Higher topological complexity and its symmetrization. Algebr. Geom. Topol., 14(4):2103-2124, 2014.

[2] J.G. Carrasquel-Vera. The rational sectional category of certain maps. To appear in Ann. Scuola Norm. Sup. Pisa Cl. Sci.

[3] J.G. Carrasquel-Vera. Computations in rational sectional category. Bull. Belg. Math. Soc. Simon Stevin, 22(3):455-469, 2015.

[4] A. Costa and M. Farber. Motion planning in spaces with small fundamental groups. Commun. Contemp. Math., 12(1):107-119, 2010.

[5] D.M. Davis, G. Dula, J. González, and M. Mahowald. Immersions of $\mathbb{R P}^{2^{e}-1}$. Algebr. Geom. Topol., 8(2):997-1030, 2008.

[6] A. Dranishnikov. The topological complexity and the homotopy cofiber of the diagonal map for non-orientable surfaces. arXiv:1506.06291v6.

[7] M. Farber. Topological complexity of motion planning. Discrete Comput. Geom., 29(2):211-221, 2003.

[8] M. Farber. Invitation to Topological Robotics. Zur. Lect. Adv. Math. European Mathematical Society (EMS), Zürich, 2008.

[9] M. Farber, S. Tabachnikov, and S. Yuzvinsky. Topological robotics: motion planning in projective spaces. Int. Math. Res. Not. IMRN, (34):1853-1870, 2003.

[10] J. González and M. Grant. Sequential motion planning of non-colliding particles in Euclidean spaces. Proc. Amer. Math. Soc., 143(10):4503-4512, 2015.

[11] J. González, B. Gutiérrez, A. Guzmán, C. Hidber, M. Mendoza, and C. Roque. Motion planning in tori revisited. Morfismos, 19(1):7-18, 2015. Available from http://www.morfismos.cinvestav.mx.

[12] J. González, B. Gutiérrez, and S. Yuzvinsky. The higher topological complexity of subcomplexes of products of spheres and related polyhedral product spaces. Topol. Methods Nonlinear Anal., in press.

[13] M. Grant, G. Lupton, and J. Oprea. Spaces of topological complexity one. Homology Homotopy Appl., 15(2):73-81, 2013.

[14] J.-C. Hausmann. Mod Two Homology and Cohomology. Universitext. Springer, Cham, 2014. 
[15] I.M. James. On the immersion problem for real projective spaces. Bull. Amer. Math. Soc., 69:231-238, 1963.

[16] J. Korbaš. Vector fields on real flag manifolds. Ann. Global Anal. Geom., 3(2):173-184, 1985.

[17] J. Korbaš and J. Lörinc. The $Z_{2}$-cohomology cup-length of real flag manifolds. Fund. Math., 178(2):143-158, 2003.

[18] K.Y. Lam. A formula for the tangent bundle of flag manifolds and related manifolds. Trans. Amer. Math. Soc., 213:305-314, 1975.

[19] G. Lupton and J. Scherer. Topological complexity of $H$-spaces. Proc. Amer. Math. Soc., 141(5):1827-1838, 2013.

[20] M. Mimura and H. Toda. Topology of Lie Groups. I, II, volume 91 of Transl. Math. Monogr. American Mathematical Society, Providence, RI, 1991. Translated from the 1978 Japanese edition by the authors.

[21] Z. Petrović and B. Prvulović. On Gröbner bases for flag manifolds $F(1,1, \ldots$, 1,n). J. Algebra Appl., 12(3):1250182, 2013, 7 pp.

[22] Y.B. Rudyak. On higher analogs of topological complexity. Topology Appl., 157(5):916-920, 2010.

[23] R.E. Stong. Immersions of real flag manifolds. Proc. Amer. Math. Soc., 88(4):708-710, 1983.

Jesús González jesus@math.cinvestav.mx

Departamento de Matemáticas, Centro de Investigación y de Estudios Avanzados del Instituto Politécnico Nacional, Av. Instituto Politécnico Nacional 2508, Col. San Pedro Zacatenco, Mexico City 07360, Mexico

Bárbara Gutiérrez bgutierrez@math.cinvestav.mx

Departamento de Matemáticas, Centro de Investigación y de Estudios Avanzados del Instituto Politécnico Nacional, Av. Instituto Politécnico Nacional 2508, Col. San Pedro Zacatenco, Mexico City 07360, Mexico

Darwin Gutiérrez dargut@hotmail.com

Departamento de Formación Básica, Escuela Superior de Cómputo del Instituto Politécnico Nacional, Juan de Dios Bátiz esq. Miguel Othón de Mendizábal, México City 07738, Mexico

Adriana Lara adriana@esfm.ipn.mx

Departamento de Matemáticas, Escuela Superior de Física y Matemáticas del Instituto Politécnico Nacional, Edificio 9, U.P. Adolfo López Mateos, Mexico City 07300, Mexico 